\section{Twin Pregnancy Complicated by Intrauterine Death of One Fetus and Herniated Bowel Loops Over Gravid Uterus}

Sir,

We present a rare case of incisional hernia with herniated bowel loops lying over gravid uterus with twin pregnancy, with need of urgent caesarean section due to death of one of the twin at 21 weeks, pre-eclampsia, and early stages of development of sepsis. Such constellation of comorbidities in a patient have not been reported before in literature and served as a dilemma for multidisciplinary teams involved in management including anesthesiologists, obstetricians and surgeons. Written informed consent was obtained from the patient for this case publication.

A 38-year old lady, gravida 5, para 3, with 31-week pregnancy, came to emergency department with complains of diarrhea (10-12 episodes per day) for 2 days. There was no history of previous antenatal visits to an obstetrician. No previous ultrasounds or any blood test report was available. She was obese with body mass index (BMI) of $35.4 \mathrm{~kg} / \mathrm{m}^{2}$. She had a history of umbilical hernia repair 10 years back, laparoscopic cholecystectomy and ventral hernia repair 8 years back, repair of large incisional hernia $(15 \mathrm{~cm})$ with mesh 3 years back, and previous 3 lower segment caesarean section (LSCS).

On examination and laboratory tests, she was diagnosed to have pre-eclampsia (raised blood pressures, headache, nausea, vomiting and significant proteinuria), high anion gap metabolic acidosis and hypokalemia. Leukocytosis and lactate level of $2.4 \mathrm{mmol} / \mathrm{L}$ was also present. Ultrasound pelvis was done, which showed mono-chorionic, mono-amniotic twins with intrauterine death of one of the twin at 21 weeks. She had a large, soft, herniated sac with multiple defects along with herniated bowell loops present over gravid uterus (Figure 1).

The patient was resuscitated, central venous catheter was inserted and electrolyte imbalance was corrected. Antibiotics were given. Surgery team was taken on board as well. Keeping in mind the maternal condition, which outweighed that of fetus, it was planned to undertake emergency caesarean section with repair of incisional hernia. Preoperatively, the patient and her husband were counselled in detail regarding her current condition and condition of her babies. Surgical plan and anesthesia options and their effect on fetus, were also discussed.

Spinal anesthesia could not be given to the patient as prolonged surgery was expected. General anesthesia could not be given because of potential difficult access to the fetus during surgery as it could have prolonged induction to delivery time and affect the APGAR of the alive fetus. Since the platelet count and international normalised ratio (INR) and prothrombin time (PT) were within normal range and there were no signs of disseminated intravascular coagulation (DIC), it was decided to give combined spinal epidural anesthesia to the patient.

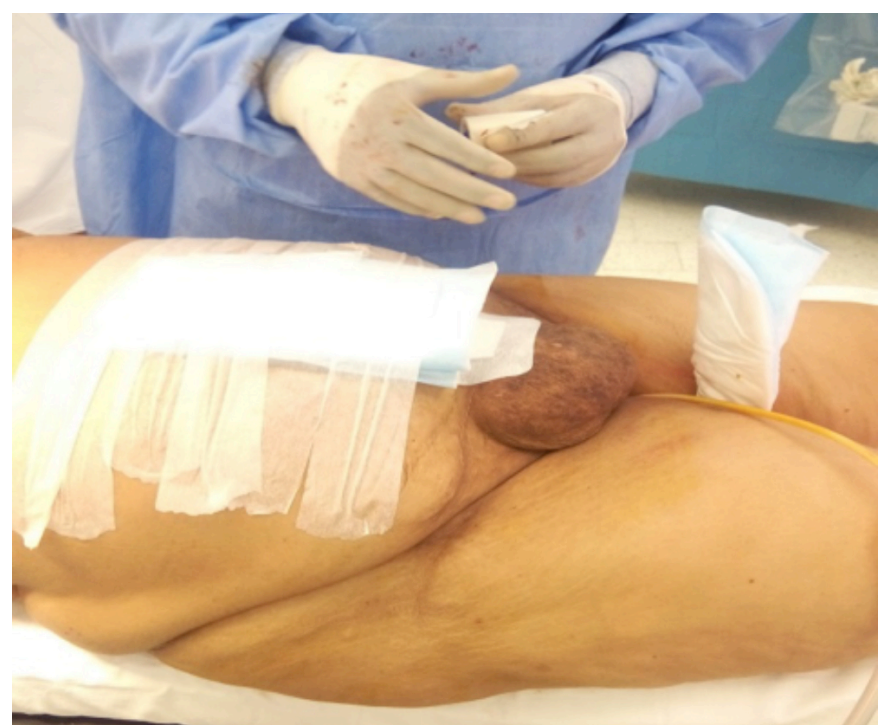

Figure 1: Postoperative picture of patient's abdomen. The hernia was planned to be repaired later. The picture shows location of existing hernia overthe uterus.

Electrocardiogram (ECG), non-invasive blood pressure monitor, and pulse oximeter were applied. Two large bore intravenous cannula of 16 gauges were inserted. Urinary catheter was already in place to monitor urine output. Patient was placed in sitting position.

The back of the patient was examined. The spinous processes and inter-spinous spaces were not palpable due to edema and obesity. The iliac crests were palpable, and at that level in the midline combine spinal epidural insertion was attempted. It was successfully inserted. $12 \mathrm{mg}$ of $0.5 \%$ hyperbaric bupivacaine with $15 \mu \mathrm{g}$ micrograms of fentanyl was given as spinal dose and the catheter was inserted in the epidural space.

Patient was placed in supine position. Surgery team did a midline laparotomy. Abdominal entry was difficult with excessive adhesions. Access to the uterus was made. Obstetric team then took over and made transverse incision on uterus. One alive and another dead fetus were delivered. Alive neonate remained well. Abdomen was closed and mesh repair was deferred till the signs of infection resolved.

During surgery, the patient remained hemodynamically stable and vomited twice during the handling of bowel loops by surgeon, which was managed with ondansetron. Epidural was loaded after the effect of spinal dose started wearing off and continued postoperatively for pain relief.

Twin pregnancy is associated with a higher morbidity and mortality as compared to singleton pregnancy. Mono-chorionic twins are at increased risk for perinatal death and neurological injury. ${ }^{1}$ Fetal demise of one twin puts the surviving co-twin at 
more risk. The prognosis is poor and associated with neurological damage in the surviving fetus. ${ }^{2}$

Pregnancy-induced hypertension and pre-eclampsia have also been found to be associated with the intrauterine death of one twin. ${ }^{3}$ Our patient had undiagnosed pre-eclampsia, which may have been the cause of intrauterine death of the fetus. Early delivery remains the definitive treatment of this condition.

Leaving the surviving co-twin in the hostile intrauterine environment is risky and should be balanced against preterm delivery problems. Conservative management is usually preferred. ${ }^{4}$

Intrauterine death may lead to maternal complications as well, which include DIC, sepsis, acute renal failure and maternal mortality. ${ }^{5}$ These complications may, in turn, cause threat to surviving twin and cause mortality.

The cause of infection in this patient was unclear. It may be due to intrauterine death, diarrhea or partial intestinal obstruction due to hernia. Nevertheless, the patient was developing early signs of sepsis and required early delivery.

General anesthesia could not be given to the patient due to expected prolonged induction to delivery time with low APGAR score in neonates. This may be due to the development of fetal acidosis as a result of longer duration of anesthesia. Also these patients may have high level of circulating catecholamines causing a reduction in placental blood flow which also leads to acidosis. Low APGAR can also be due to transmission of anesthetic drugs though placental barrier.

Regional anesthesia may be regarded as superior to general anesthesia for caesarean section. In this patient, spinal anesthesia could not be given due to prolonged duration of surgery, so combined spinal epidural anesthesia was selected. It not only provided anesthesia intraoperatively, but was continued postoperatively foranalgesia. In pre-eclamptic patients, neuraxial anesthesia actually provides more stable hemodynamics with fewer swings in blood pressure.

The case was successfully managed with no complications; and the mother and living baby remained well postoperatively.

\section{CONFLICT OF INTEREST:}

The authors declared no conflict of interest.

\section{AUTHORS' CONTRIBUTION:}

MH: Conceptualisation; formal analysis; methodology; project administration; supervision; validation; visualisation; writing original draft; writing review and editing

$\mathrm{KA}$ : Conceptualisation; data curation, validation; visualisation; writing review and editing

\section{REFERENCES}

1. Cheong-See F, Schuit E, Arroyo-Manzano D, Khalil A, Barrett J, Joseph KS, et al. Prospective risk of stillbirth and neonatal complications in twin pregnancies: Systematic review and meta-analysis. BMJ 2016; 354:i4353.

2. Mackie FL, Morris RK, Kilby MD. Fetal brain injury in survivors of twin pregnancies complicated by demise of one twin: A review. Twin Res Hum Genet 2016; 19(3): 262-7.

3. Yuan T, Wang W, Li XL, Li CF, Li C, Gou WL, et al. Clinical characteristics of fetal and neonatal outcomes in twin pregnancy with preeclampsia in a retrospective case: Control study: A strobe-compliant article. Medicine 2016; 95(43): e5199.

4. Woo $H$, Sin S, Tang L. Single foetal death in twin pregnancies: Review of the maternal and neonatal outcomes and management. Hong Kong Med J 2000; 6(3): 293-300.

5. Patel $\mathrm{S}$, Thaker R, Shah P, Majumder S. Study of causes and complications of intra-uterine fetal death (IUFD). Int J Reprod Contracept Obstet Gynecol 2014; 3(4):931-5.

Malika Hameed and Khalid Ahsan

Department of Anesthesiology, The Aga Khan University Hospital, Karachi, Pakistan

Correspondence to: Dr. Malika Hameed, Department of Anesthesiology, The Aga Khan University Hospital, P.O. Box 3500, Stadium Road, Karachi, Pakistan

E-mail: malika.hameed@gmail.com

Received: March 13, 2019; Revised: August 07, 2019;

Accepted: September 12, 2019

DOI: https://doi.org/10.29271/jcpsp.2020.06.669 